\title{
Gradient sample argument weighting for robust image region description
}

\author{
John-Olof Nilsson and Peter Händel \\ Signal Processing Lab, ACCESS Linnaeus Centre, KTH Royal Institute of Technology, Stockholm, Sweden
}

\begin{abstract}
The weighting of gradient sample arguments for the creation of descriptors of image regions is studied. The descriptors are interpreted as binned and weighted argument kernel density estimates and thereby their defining attributes are identified as the binning rules and the weighting. The weighting is further studied and four different weighting strategies are analyzed. The naive constant weighting is argued to have a poor robustness to image perturbations. As an answer to this, the customary gradient magnitude weighting is motivated. However, the short-comings of this approach are pointed out and two novel weighting strategies are suggested. The first suggested weighting gives a system parameter determining a distinctiveness to robustness trade-off with the customary magnitude weighting being a special case of it. The second suggested weighting gives a similar robustness as the first one, but at a lower computational cost. Finally, the effects of the different weighting strategies are demonstrated with real imagery data and synthetic perturbations.
\end{abstract}

\section{INTRODUCTION}

Many popular computer vision algorithms exploit gradient sample argument histograms to describe regions in an image, e.g. [1][2][3]. Such histogram can be interpreted as argument density estimates. However, the situation is rather different when it is compared with a normal density estimation. This is because the density estimates are arbitrary as long as they are distinct and robust to perturbations in the image domain. In other words, the estimates themselves do not matter but, rather their ability to robustly describe the region of their origin. Consequently, we might weight the gradient sample arguments arbitrarily to achieve this. The gradient sample argument distributions, which give the density estimates, are empirical distributions. However, the image and therefore also the gradient samples have stochastic as well as systematic disturbances due to perspective changes, changing lighting conditions, camera parameter settings, etc. Unfortunately, the effects of the disturbances are different for different gradients. Therefore, to make the distribution and density estimates robust to such disturbances, different gradient samples should be treated differently. Normally, the gradient sample arguments are weighted with the magnitude of the gradient, giving a weighted histogram and a corresponding weighted density estimate. This makes the density estimate distinct and robust to image perturbations. However, while simple and generally used in the field, this weighting is motivated nowhere and, as we will show, has its short-comings. Therefore, in this article we discuss the gradient sample weighting and suggest two novel weighting strategies with improved robustness properties. The robustness is illustrated with real imagery data and synthetic perturbations. Any method using a gradient sample histogram can easily incorporate these novel weighting strategies, so they are therefore believed to have a wide applicability.

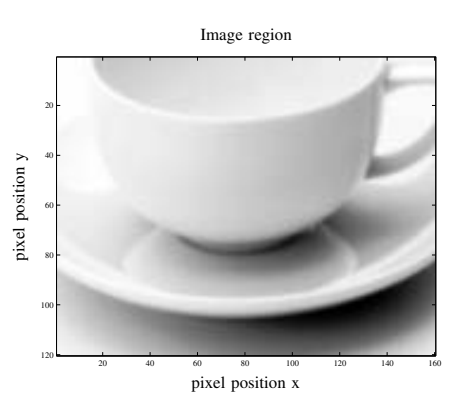

(a)

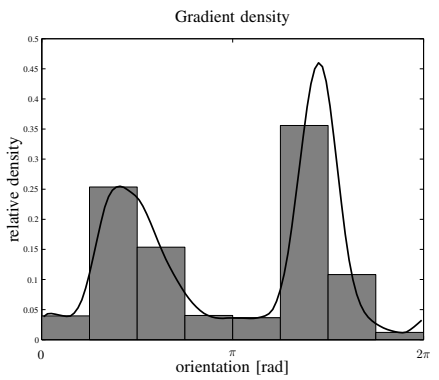

(b)
Fig. 1: The figures show an image region (a) and the corresponding gradient argument histogram (b) with magnitude weighting and a linear binning. Overlaid is the continuous kernel density estimate given by a triangular kernel with a width of $22.5\left[^{\circ}\right]$.

\section{IMAGE REGION DESCRIPTION AND DENSITY ESTIMATION}

To create a descriptor of an image region, gradient samples from the region are divided into different orientation groups and weighted by their magnitude, i.e. a weighted gradient argument histogram is created [1][2][3]. The creation of the histogram can be formalized as follows. From a set of gradient samples $\nabla s_{k} \in \mathbb{R}^{2}$ indexed by $k$, the gradient argument histogram bin $h_{i}$ of the histogram $h$ is determined by

$$
h_{i}=\sum_{k} w\left(\nabla s_{k}\right) \delta_{i}\left(\arg \left(\nabla s_{k}\right)\right)
$$

where $w(\cdot)$ is some weight, $\delta_{i}(\cdot)$ is the binning rule, and $\arg (\cdot)$ is the gradient argument/phase. Most often the weight $w(\cdot)=$ $\|\cdot\|$ and a simple or a $2 \pi$-modular linear binning rule are used. See [4] for definitions and a detailed treatment of binning rules. Typically the weight $w(\cdot)$ is normalized such that $\sum_{i} h_{i}=1$.

The histogram creation (1) can be mapped to the kernel density estimation [4][5] and the binning will simply introduce a sampling of the continuous kernel density estimate. Consequently, we can assess the effects of $w(\cdot)$ and $\delta_{i}(\cdot)$ in the continuous case without the effect of binning. In Fig. 1 an image region and a corresponding 8-bin histogram are shown. The histogram makes up the descriptor of the image region. Overlaid is the continuous density estimate of the gradient argument distribution created with a triangular kernel with the width $360 / 8=22.5{ }^{\circ}$ ] (corresponding to linear binning). Note that the descriptor/binning corresponds with the sampling of the continuous density estimate. From (1) it is easily seen that the density estimation is defined by the weight $w(\cdot)$ and the binning rule $\delta_{i}(\cdot)$. We shall be concerned with the former. Note that often a second spatial weight is added to mitigate 
the effect of any variation in the described image region position/boundaries. However, this weight is independent of $w(\cdot)$ and $\delta_{i}(\cdot)$ and therefore, in this article, we will ignore the spatial weighting. In the following subsections four different weights $w()$ and their characteristics will be discussed. To facilitate the discussion, we will factor the weight as

$$
w(\cdot)=\rho w_{\star}(\cdot)
$$

where $\rho$ is a normalizing constant ensuring that $\sum_{i} h_{i}=1$ and $w_{\star}(\cdot)$ gives the relative weighting, where $\star$ is an index indicating one of the weights discussed below. All of the weights are illustrated in Fig. 2.

\section{A. Constant weighting}

The simplest weight we can use for the gradient sample arguments is a constant weight, i.e.

$$
w_{c}\left(\nabla s_{k}\right)=c
$$

where $c$ is an arbitrary constant [1]. A gradient argument density estimate with this weighting will essentially give a measurement of how large an area, in the described image region, there is that has a gradient in a certain direction. Every area (every sample) will have an equal contribution. This ensures a density estimate with many features. Unfortunately, the gradient orientation of smooth regions may change abruptly with changing lighting conditions and sharp gradients may disappear under a large amount of smooth gradients. Therefore, as we will argue in the next section, and see in Section III, this gives a gradient density estimate which is sensitive to image perturbations.

\section{B. Magnitude weighting}

When sampling gradients, apart from the argument, the gradient magnitude will also be available. This magnitude provides more information about an argument sample which can be used to make the gradient argument density more distinct. With no weighting, two samples with the same argument but with (vastly) different magnitudes will give the same contribution to the density estimate. This means that many combinations of gradient samples are mapped to the same density estimate. This is avoided by adding a monotonically increasing weight as a function of the gradient magnitude. The gradient magnitude is independent of image rotation and therefore the weight should be a function of it. Also, to make the weighting independent of the image intensity we want

$$
w_{\star}\left(\nabla\left(a \cdot s_{k}\right)\right)=a w_{\star}\left(\nabla s_{k}\right)
$$

such that $w\left(\nabla\left(a \cdot s_{k}\right)\right)$ becomes independent of $a$. This is fulfilled by weighting with the gradient magnitude itself, i.e.

$$
w_{m}\left(\nabla s_{k}\right)=\left\|\nabla s_{k}\right\| \text {. }
$$

Another attribute of this weighting is that it is independent of the scale of an inspected image patch. This is since the gradients will scale with the zoom, but a proportionally greater number of samples will be achieved. The weight (4) will also give an argument density which is more robust to disturbances compared with weight (2). A large gradient will be more robust to image perturbations than smaller gradients. Assume that the 'true' gradient $\nabla s_{k}$ is perturbed with $\left[\epsilon_{\|} \epsilon_{\perp}\right]$ where $\epsilon_{\|}$is the perturbation parallel and $\epsilon_{\perp}$ is the perturbation perpendicular to the gradient. Then the resulting perturbation $\epsilon_{\angle}$ of the gradient argument is

$$
\epsilon_{\angle}=\operatorname{atan} 2\left(\left\|\nabla s_{k}\right\|+\epsilon_{\|}, \epsilon_{\perp}\right)
$$

where $\operatorname{atan} 2(\cdot, \cdot)$ is the four quadrant (two argument) arctangent function. If the perturbation is small compared to the gradient magnitude then $\operatorname{atan} 2\left(\left\|\nabla s_{k}\right\|+\epsilon_{\|}, \epsilon_{\perp}\right) \approx \frac{\epsilon_{\perp}}{\left\|\nabla s_{k}\right\|+\epsilon_{\|}}$ and

$$
\epsilon_{\angle} \approx \frac{\epsilon_{\perp}}{\left\|\nabla s_{k}\right\|+\epsilon_{\|}} \approx \frac{\epsilon_{\perp}}{\left\|\nabla s_{k}\right\|} .
$$

For large gradient magnitudes, due to the statistical linearity of (5), this implies that $\epsilon_{\angle}$ is distributed approximately as $\frac{1}{\left\|\nabla s_{k}\right\|} \epsilon_{\perp}$ and that

$$
\operatorname{std}\left(\epsilon_{\angle}\right) \approx \frac{1}{\left\|\nabla s_{k}\right\|} \operatorname{std}\left(\epsilon_{\perp}\right),
$$

where $\operatorname{std}(\cdot)$ denotes the standard deviation. In other words, the standard deviation of the gradient argument is inversely proportional to the gradient magnitude. From the distribution estimation point of view, $\operatorname{std}\left(\epsilon_{\angle}\right)$ gives a measure of the likelihood that the argument sample is contributing to the right bin in the gradient argument histogram, i.e. the weighting (4) is roughly proportional to the quality of the gradient sample. Therefore, the weighting (4) gives a density estimate which is robust to perturbations. Consequently, with weight (4) we get a more distinct and robust argument density estimate when compared with (2) and the weight is independent of the image scale, intensity, and rotation.

\section{Magnitude weighting with threshold}

The problem with weighting with a gradient magnitude is that (6) only holds for large gradients and the manifold nature of the argument means that the standard deviation is only a sensible measure of the perturbation of the argument for small perturbations. Also, the image perturbations are often not zero-mean and spatially white. For example, changing lighting conditions may give a systematic bias of the gradients and an argument bias which is inversely proportional to the gradient magnitude. The gradient magnitude can be seen as the signal power, and consequently the magnitude weighting can be thought of as reasonable only in a high signal-to-noise regime.

Unfortunately, an image, especially of a man-made environment, can have significant portions of smooth and evenly reflective surfaces. Gradient samples from such areas will be small, and therefore, the magnitude weighting will give undesirable effects for large portions of many scenes. Consequently, gradient samples with a small magnitude may corrupt the density estimate and we wish to ignore these completely. This suggests that it is necessary to use a threshold on the magnitude weighting. However, with just a truncation of the weight (4) as briefly mentioned in [6], small perturbations could give large changes in the weight. Therefore, we also subtract the threshold itself from the gradient magnitude giving the weight

$$
w_{t}\left(\nabla s_{k}\right)=\left\{\begin{array}{lll}
0 & \text { if } & \left\|\nabla s_{k}\right\|<\gamma \\
\left\|\nabla s_{k}\right\|-\gamma & \text { if } & \left\|\nabla s_{k}\right\| \geq \gamma
\end{array}\right.
$$

In essence this will guard against perturbations which are significantly smaller than the threshold $\gamma$. Since $\gamma=0$ makes 
(7) equivalent to (4), the former can be seen as a generalization of the latter. Obviously, (7) will not be independent of image intensity and scale. However, for the distinct gradient components and a reasonable threshold $\left\|\nabla s_{k}\right\| \gg \gamma$, the weight will be approximately independent of intensity and scale for the 'interesting' samples. Also, the lack of scale independence may also make it more distinct since it will distinguish between differently sharp gradients regions.

A similar type of thresholding of the density estimates themselves has been suggested in [1]. This will suppress a base noise level in the density estimates. This thresholding is unfortunate for three reasons. 1) It does not guard against weak lighting gradient which might sum up to significant peaks in the distribution while potentially undergoing large changes due to lighting variations. 2) Simple thresholding of the density estimate may produce abrupt jumps in the density estimates, when a bin $h_{i}$ is around the threshold, and a cross-coupling between samples. 3) Evaluation of the binning carries a significant computational cost. Thresholding the distribution estimate means that all of the samples will have to be binned independently if they contribute to the final thresholded estimate. In contrast with (7), only samples with a non-zero weight will have to be binned. For man-made environments, this can provide a substantial computational cost saving.

\section{Quadratic weighting}

To make the weighting rotationally invariant, the weight needs to be a function of the gradient magnitude. However, the attribute (3) which gave (4) is not holy. The important quality is that we can make the distribution estimate invariant to the image intensity with the normalization $\rho$. This means that in general the weight should be homogeneous, i.e.

$$
w\left(\nabla\left(a \cdot s_{k}\right)\right)=a^{p} w\left(\nabla s_{k}\right)
$$

where $p$ is a constant. Further, the improved distinctiveness only requires a monotonous weight. If two gradient sample from different images related to the same point in the scene are both subject to independent perturbations, the chance that they will both contribute to the same bin is roughly proportional to the variance rather than the standard deviation. Finally, the gradient magnitudes as used in (7) and (4),

$$
\left\|\nabla s_{k}\right\|=\sqrt{\left(\nabla_{x} s_{k}\right)^{2}+\left(\nabla_{y} s_{k}\right)^{2}},
$$

contain a square-root and are therefore simply expensive to calculate. A linear weighting with the magnitude may be approximated with projections but for few bins the squareroot has to be calculated. This suggests that we should use a quadratic weight. The quadratic weight is obviously homogeneous and proportional to the variance and eliminates the square root. In this case, the thresholding as of (7) fills less of a function to suppress small error prone gradients. However, the thresholding may still give a significant computational cost reduction and no thresholding is achieved as a special case with the threshold equal to zero. Therefore, the thresholding is retained giving the weight

$$
w_{q}\left(\nabla s_{k}\right)= \begin{cases}0 & \text { if }\left\|\nabla s_{k}\right\|^{2}<\gamma^{2} \\ \left\|\nabla s_{k}\right\|^{2}-\gamma^{2} & \text { if }\left\|\nabla s_{k}\right\|^{2} \geq \gamma^{2}\end{cases}
$$

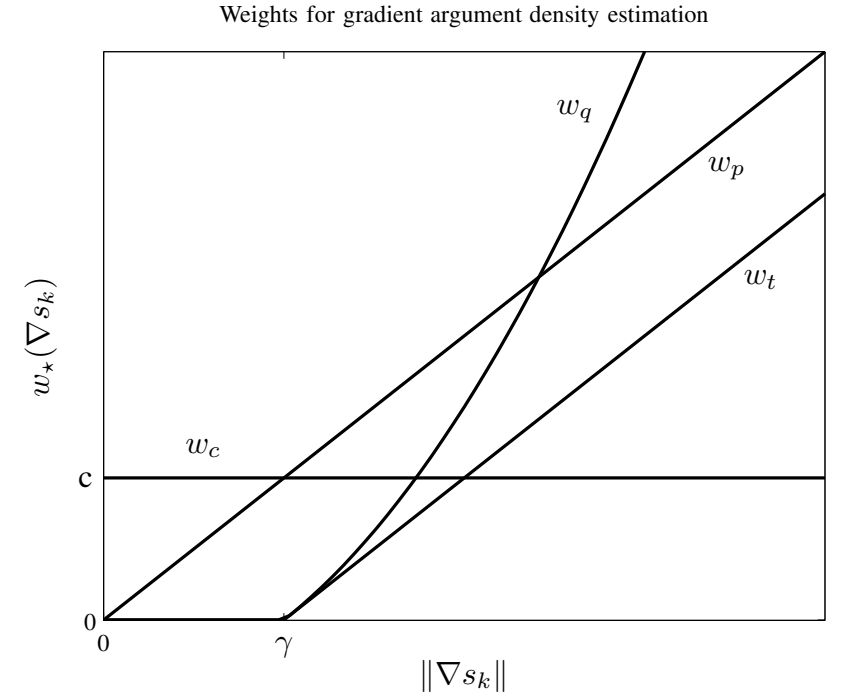

Fig. 2: Illustrations of the four suggested weights $w_{c}, w_{p}, w_{t}$, and $w_{q}$ as a function of the gradient magnitude.

The main merit of this weight is the lower computational cost resulting from the elimination of the square root in the gradient sample magnitude. Also, similar to the magnitude weighting with threshold, it will distinguish between differently sharp gradient regions. However, it has two notable draw-backs. 1) Due to the quadratic dependence (homogeneity of degree two) on the image intensity, it may be more sensitive to nonlinear intensity changes, e.g. camera gain non-linearities, nondiffusive reflection, color dependence of camera gain, etc., compared with a weight with a homogeneity degree of one. 2) If there are few large gradients, the distinctiveness may suffer since the corresponding density estimate will be dominated by a few large peaks. However, this sparsity may also be exploited by compressed sensing techniques and to compress the descriptors.

\section{EXPERIMENTAL RESULTS}

In the preceding sections, four different weights have been discussed. These weights are illustrated in Fig. 2. To demonstrate the effects of the different weights, in this section we exemplify argument distribution estimates resulting from the different weights for images with synthetic perturbations. To achieve a reference image and reference distribution estimates, a high resolution image was low-pass filtered with a Gaussian kernel and subsampled to create the essentially noise free image patch shown in Fig. 1a. From this reference image, two perturbed images were created, one shown in Fig. 3a with a lighting gradient in orientation $\pi$ added to it and one shown in Fig. $3 \mathrm{~b}$ with a $35[\mathrm{~dB}]$ PSNR (peak signal-to-noise ratio) image noise added to it. From the images, weighted gradient distribution estimates with the different weights were calculated. To stress the effects of the weighting, no binning was used but a continuous density estimates were calculated with a $2 \pi$-modular Gaussian kernel with a bandwidth of $3\left[^{\circ}\right]$. The threshold $\gamma$ was set to four standard deviations of the image noise. The kernel density estimates are seen in Figs. 3c-f.

The lighting gradient adds a bias toward orientation $\pi$ of 


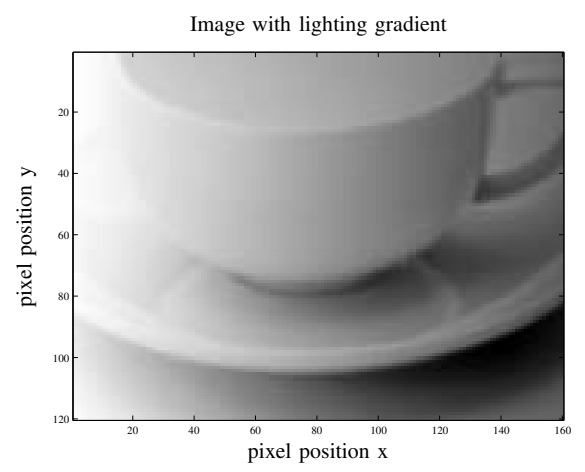

(a) Image with lighting gradient added corresponding to the dotted lines in adjacent plots.

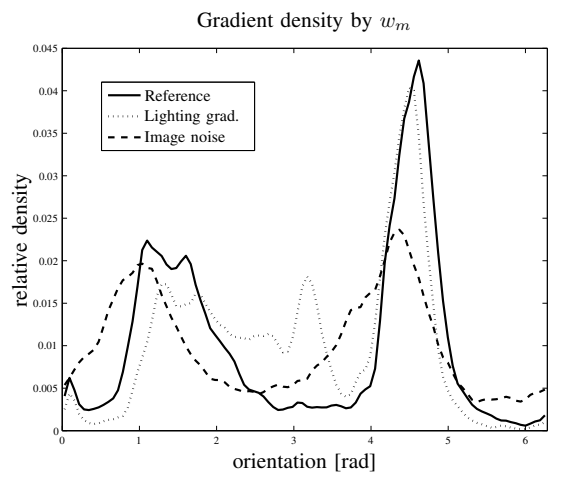

(d) Argument density estimates with weighting with $w_{m}$.

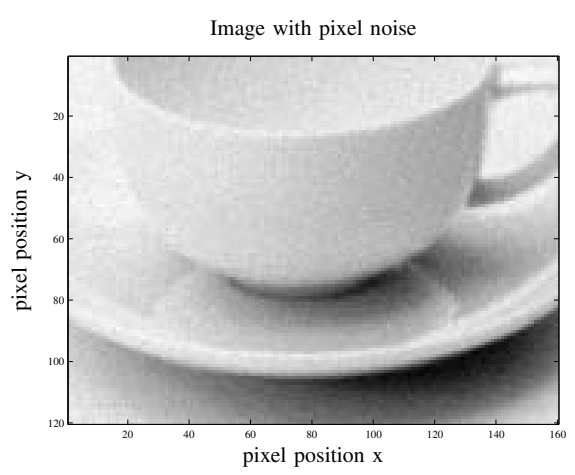

(b) Image with pixel noise of $35[\mathrm{~dB}]$ PSNR corresponding to dashed lines in adjacent plots.

Gradient density by $w_{t}$

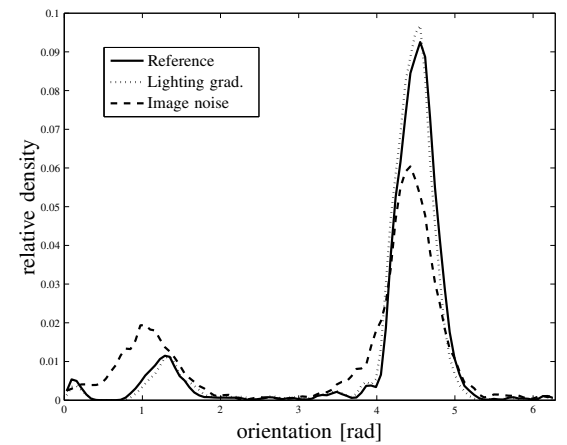

(e) Argument density estimates with weighting with $w_{t}$.

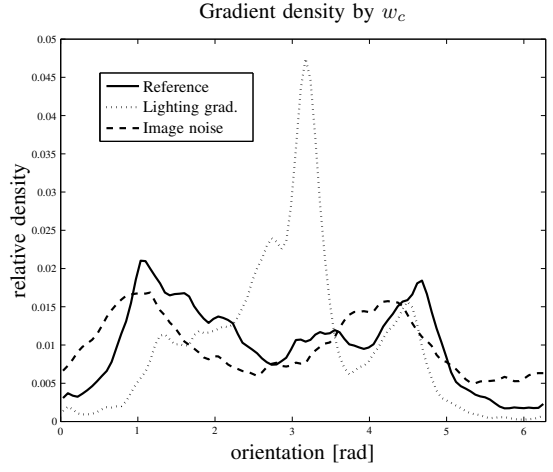

(c) Argument density estimates with weighting with $w_{c}$.

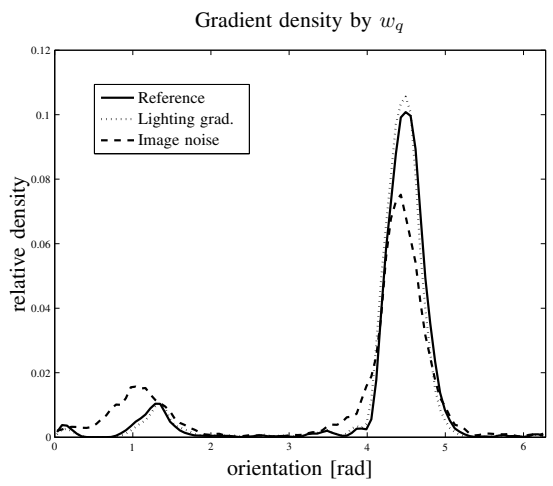

(f) Argument density estimates with weighting with $w_{q}$.

Fig. 3: Subfigures (c)-(f) show argument density estimates of the reference image in Fig. 1a (solid lines), the reference image with an added lighting gradient (a) (dotted lines), and the reference image with a 35[dB] PSNR image noise (b) (dashed lines). The gradients were calculated by differences of adjacent pixel values for all image pixels. Clearly, $w_{c}$ provides the worst robustness. The magnitude weighting $w_{m}$ performs better but significant changes are still seen. Both $w_{t}$ and $w_{q}$ show good robustness to the image perturbations.

all gradient arguments. This is seen around $\pi$ in the argument density estimate weighted by $w_{c}$ (Fig. 3c) and to a lesser extent in that weighted by $w_{m}$ (Fig. 3d) while the effects on the density estimates weighted by $w_{t}$ and $w_{q}$ (Fig. 3e-f) are seen to be marginal. The image noise has the consequence of scattering the contributing components to the density estimate. This effect is noticeable in all density estimates but to a higher degree in those weighted by $w_{c}$ and $w_{m}$. The results clearly show that a constant weighting gives a description of the image with an inadequate robustness. However, significant changes are also seen in the density estimates for the customary magnitude weighting. These are almost eliminated by the truncated and quadratic weights. The improved robustness is essentially achieved by rejecting easily corrupted gradient samples.

\section{CONCLUSION}

In this article we have pointed out the equivalence of the creation of gradient sample argument histograms for image region description with a weighted kernel density estimation. Based on this, the defining attributes have been recognized to be the binning rule and the weighting. A constant, the customary and two novel weighting strategies have been discussed and their robustness properties analyzed. Finally, synthetic perturbations of an image have been used to demonstrate that an improved robustness is achieved with the novel strategies.
For scenes with a significant portion of smooth and evenly reflective surfaces, the magnitude weighting with a threshold will probably give more robust density estimates compared with the customary magnitude weighting. However, the exact performance characteristics and trade-offs between the weights will be dependent on the application and both remain to be tested in a practical scenario.

\section{REFERENCES}

[1] D. G. Lowe, "Distinctive image features from scale-invariant keypoints," International Journal of Computer Vision, vol. 60, pp. 91-110, 2004.

[2] N. Dalal and B. Triggs, "Histograms of oriented gradients for human detection," in International Conference on Computer Vision \& Pattern Recognition, pp. 886-893, June 2005.

[3] K. Mikolajczyk and C. Schmid, "A performance evaluation of local descriptors," IEEE Trans. PAMI, vol. 27, pp. 1615 -1630, oct. 2005.

[4] P. Hall and M. Wand, "On the accuracy of binned kernel density estimators," Journal of Multivariate Analysis, vol. 56, pp. 165-184, 1996.

[5] B. Wang and X. Wang, "Bandwidth selection for weighted kernel density estimation," Electronic Journal of Statistics, 2011. arXiv:0709.1616.

[6] D. G. Lowe, "Object recognition from local scale-invariant features," in Proc. of the Inertnational Conference on Computer vision, 1999. 